\title{
Interhemispheric transfer of test stimulus representations in memory scanning*t
}

\author{
ROBERTA L. KLATZKY \\ Stanford University, Stanford, Calif. 94305
}

Test stimuli were presented in either the right or left visual field during a memory scanning task. The Ss indicated whether or not a given test stimulus, either a letter or a picture, matched one of a previously presented set of letters. Reaction times were recorded and plotted as a function of the size of the set of letters. The results are in agreement with the hypothesis that the two types of test stimuli are processed by different cerebral hemispheres with differing stimulus representations.

On the basis of their work with human Ss whose cerebral commissures had been surgically separated, Levy-Agresti \& Sperry (1968) have hypothesized that the left speech hemisphere and the right hemisphere differ in their modes of information processing. The left hemisphere appears to be analytic, whereas the right processes information in terms of its whole configuration. Accordingly, the right hemisphere excels at problems that require more Gestalt, spatial processing, and the left hemisphere "processed stimulus information in such a way that the stimulus could be described in language [Levy, 1969, p. 614]." In short, the right and left hemispheres might be designated "spatial" and "verbal" processors, respectively.

The results of several experiments investigating differences in information processing between the two cerebral hemispheres tend to support this hypothesis (e.g., Filbey \& Gazzaniga, 1969; Gibson, Filbey, \& Gazzaniga, 1970; Moscovitch \& Catlin, 1970). In these studies stimuli were presented to either the right or left hemisphere and reaction times (RT) were recorded. The resulting data indicate that RT is greater when information must be transmitted across the corpus callosum in order for a response to be made than when such a callosal transmission is unnecessary. The increase in $\mathrm{RT}$ must be attributed in some way to the information transfer.

Filbey \& Gazzaniga (1969) and Gibson et al (1970) required Ss to make a verbal response to a stimulus presented in either the right or the left visual field. Since the left hemisphere elicits the verbal response,

\footnotetext{
*This research was supported by the National Aeronautics and Space Administration, Grant No. NGR-05-020-244.

tThe author gratefully acknowledges the contributions of Professors R.C. Atkinson and C. R. Hamilton to this experiment; they have been of great assistance in all phases of this research. Thanks are also due to J. F. Juola for programming the experimental apparatus and assisting with the data analy sis.
}

RT was less when the information was presented to the right visual field than when it appeared on the left. It is assumed that in the latter case the information entered the right hemisphere and had to be transferred across the callosum before a response could be made. Similar results were also obtained by Moscovitch \& Catlin (1970). In addition, Gibson et al found that when the verbal response was dependent on the comparison of two spatial configurations, the intial RT difference reversed, suggesting that the right hemisphere processed the stimuli.

The characterization of the two hemispheres as spatial and verbal is of special interest in connection with two recent studies using a memory scanning paradigm (Klatzky \& Atkinson, 1970; Klatzky, Juola, \& Atkinson, 1970). These experiments consisted of a series of trials of the following nature: On each trial a set of letters (the memory set) was presented to $S$ to be maintained in short-term memory; the size of this set varied from trial to trial. Then $S$ was presented with a test stimulus and required to indicate manually whether that test stimulus matched one of the members of the memory set. On some trials the test stimulus was a letter, in which case $S$ made a positive response only if that letter was contained in the memory set. On other trials, however, the test stimulus was a picture of some common object. On such a trial S answered positively only if the first letter of the name of that picture was a member of the memory set. RT was recorded and functions relating RT to memory set size were plotted.

Sternberg (1966) has formulated a theory of memory scanning which divides the scanning process into three stages. In the first, the test stimulus is processed and transformed into a representation comparable to the memorized set of letters. During Stage 2, the test-stimulus representation is compared serially to members of the memory set. In the third stage, $\mathrm{S}$ makes a decision and responds according to the results of the comparison process. If the comparisons are terminated and a response is made immediately after matching the test stimulus with a memory set element, the scanning process is called self-terminating. If $\mathrm{S}$ initiates a response only after comparing the test stimulus with every member of the memory set, regardless of whether or not a match has been made, the process is exhaustive. In terms of the reaction-time functions, the slope of the function for negative responses represents the time required to make a single comparison during Stage 2, while the intercept of either function corresponds to the time devoted to Stages 1 and 3. If the process is exhaustive, the slope of the function for positive responses should be precisely the same as for negatives, but if the process is self-terminating, the ratio of the slope for negatives to the slope for positives should to $2: 1$.

On the basis of previous research (Klatzky \& Atkinson, 1970; Klatzky et al, 1970), it has been hypothesized that letter test stimuli are treated as spatial objects in this memory scanning task and compared to the memory set in terms of their physical configuration. Pictures, in contrast, are supposed to be processed verbally in these experiments, with the comparisons made between verbal labels (e.g., "ell" for L, etc.). If this is true, it is quite possible that the processing of letter test stimuli is performed by the right cerebral hemisphere, while the left hemisphere processes the picture test stimuli. This hypothesis predicts that the presentation of a picture test stimulus to the right hemisphere (i.e., in the left visual field) should require a longer Stage 1 processing time than should a left-hemispheric presentation, with reverse effects for letter test stimuli (the increased time corresponding to the transfer of the stimulus to the appropriate hemisphere). Therefore, the intercept of the RT function for pictures should be greater for right-hemispheric presentation than for left, and the intercept of the function for letter test stimuli should be greater for left-hemispheric presentation than for right. If the comparison process of Stage 2 is unaffected by callosal transmission, the slopes should be unaffected by presentation to one or the other hemispheres. In order to make a preliminary test of these predictions, the present experiment was conducted.

$$
\text { METHOD }
$$

The Ss were eight right-handed females enrolled at Stanford University and other colleges in the area. Each $S$ was paid $\$ 10.00$ for her participation in one training session and four additional test sessions of $1 \mathrm{~h}$ each. 
Table 1

Reaction Times in Msec for Each Memory Set Size and Slope and Intercept Values in Msec for the Reaction-Time Functions for Each Response Type. Test Stimulus Type. and Hemisphere of Test Stimulus Presentation

\begin{tabular}{|c|c|c|c|c|c|c|c|c|}
\hline & & & \multicolumn{4}{|c|}{ Size of Memory Set } & \multirow[b]{2}{*}{ Slope } & \multirow{2}{*}{$\begin{array}{l}\text { Inter- } \\
\text { cept }\end{array}$} \\
\hline & & & 2 & 3 & 4 & 5 & & \\
\hline \multirow{4}{*}{$\begin{array}{l}\text { Right } \\
\text { Hemisphere } \\
\text { Presentation }\end{array}$} & \multirow{2}{*}{ Letter } & $\begin{array}{l}\text { Negative } \\
\text { Response }\end{array}$ & 824.7 & 894.9 & 951.8 & 986.7 & 54.3 & 724.5 \\
\hline & & $\begin{array}{l}\text { Positive } \\
\text { Response }\end{array}$ & 718.2 & 779.5 & 844.4 & 862.2 & 49.7 & 627.1 \\
\hline & \multirow{2}{*}{ Picture } & $\begin{array}{l}\text { Negative } \\
\text { Response }\end{array}$ & 964.3 & 1016.8 & 1078.5 & 1113.6 & 51.0 & 864.8 \\
\hline & & $\begin{array}{l}\text { Positive } \\
\text { Response }\end{array}$ & 830.7 & 864.6 & 886.8 & 947.3 & 37.2 & 752.2 \\
\hline \multirow{4}{*}{$\begin{array}{l}\text { Left } \\
\text { Hemisphere } \\
\text { Presentation }\end{array}$} & \multirow{2}{*}{ Letter } & $\begin{array}{l}\text { Negative } \\
\text { Response }\end{array}$ & 875.6 & 941.7 & 981.5 & 1046.7 & 55.3 & 767.8 \\
\hline & & $\begin{array}{l}\text { Positive } \\
\text { Response }\end{array}$ & 758.9 & 813.6 & 870.8 & 891.6 & 45.5 & 674.5 \\
\hline & \multirow{2}{*}{ Picture } & $\begin{array}{l}\text { Negative } \\
\text { Response }\end{array}$ & 950.1 & 1013.6 & 1119.1 & 1143.2 & 68.5 & 8.16 .7 \\
\hline & & $\begin{array}{l}\text { Positive } \\
\text { Response }\end{array}$ & 815.4 & 904.5 & 919.0 & 972.1 & 48.5 & 733.0 \\
\hline
\end{tabular}

The experimental apparatus was an Iconix automated tachistoscope and exposure box. The stimuli were slides presented to $S$, centered in a circular viewing area of 2-1/16-in. diam, at a line-of-sight viewing distance of approximately $2 \mathrm{ft}$.

Responses were made with three telegraph keys arranged in an arc on a table in front of S. One-half of the Ss used their right forefingers to operate these keys, while the rest used their left. (This procedure was used to balance possible effects of the pathways between hand and brain, as in Bradshaw \& Perriment, 1970.) Within these two groups, one-half of the Ss pressed the key on the right for a positive response and the key on the left for a negative response; the others used the reverse arrangement. The $S$ was instructed to depress the center key before the test and to release it only when she was ready to respond.

At the beginning of each session, $S$ viewed and named the 54 black-and-white drawings that were to be used as picture test stimuli before she began her series of 160 trials. At the onset of each trial, a memory set of two to five letters appeared. The $S$ looked directly at the set until she had memorized it, and then turned it off by pushing a button held in her nonresponse hand. About $5 \mathrm{sec}$ later, a light flashed below the viewing area, and an auditory signal of two clicks indicated to $S$ that she could begin the test. The $S$ then fixated on one of two small dots, located 2 in. to the right and 2 in. to the left of the center of the viewing area, and again pressed the button. After $500 \mathrm{msec}$, the test stimulus (a letter or a picture) appeared on the screen for $400 \mathrm{msec}$. fixation during tests remained constant within a block, while both letter and picture test stimuli appeared in a random sequence from trial to trial. The direction of fixation alternated between blocks. according to E's instructions.

\section{RESULTS}

The data analysis includes Sessions 2-5: the first training session was omitted. Only the data from correct responses were analyzed; however, the error rate for each $S$ was low, and error rate did not appear to be affected by direction of fixation. (The mean error rate was $3.3 \%$ : the range over Ss was $2.0 \%-4.5 \%$.) In addition, the first six trials of each test session were considered warmup trials and their data were also excluded from the analysis.

In Table 1, the RTs for each memory set size, test stimulus type, response type, and hemisphere of presentation are given, averaged over Ss. The theory of memory scanning predicts a linear relationship between RT and memory set size, and linear RT functions determined by the method of least squares provide a good fit to the data of this experiment. The table presents the slopes and intercepts of these functions for each condition.

Figure I shows the RT functions for each type of test stimulus and for each hemisphere, where the data for positive and negative responses have been combined. The slopes and intercepts of the best-fitting straight lines are also indicated in the figure.

\section{DISCUSSION}

The data of this experiment are, on the whole, quite similar to other memory scanning studies of this type (Klatzky \& Atkinson, 1970; Klatzky et al, 1970), indicating that the peripheral presentation of stimuli does not affect the nature of memory search. For both positive and negative responses and for both left and right fixation, the RT functions are linear and increasing. As in previous research, the

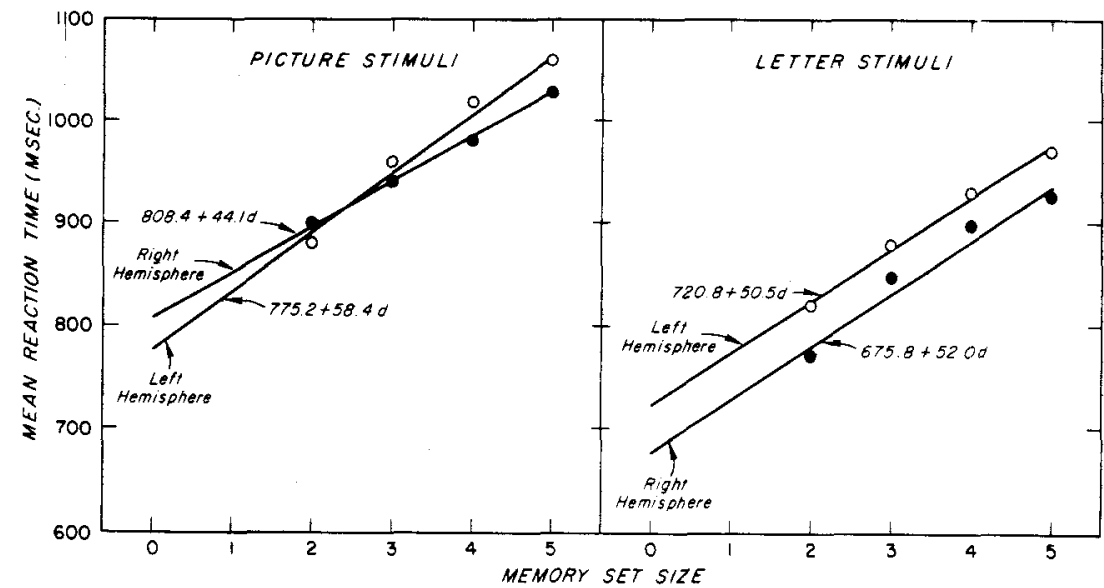

Fig. 1. Reaction time in milliseconds of responses to picture (left panel) and letter (right panel) stimuli presented to the right or left hemisphere, as a function of memory set size (d), averaged over Ss and positive and negative responses. 
ratio of the slope of the function for negative responses to the slope for positives is close to unity in the case of letters and somewhat greater (about 1.4) for picture stimuli: these slope ratios are essentially the same for both left and right fixation. These data also resemble the previous results in that the serial-position functions (which relate RT for a correct positive response to the position of the test stimulus in the memory set) are increasing; this also occurs for both left and right fixation.

One effect of peripheral presentation appears to be an increase in the slope of the RT functions for letters, relative to the slope for pictures. Previously, it has been found that the slope for pictures is greater than the slope for letters, indicating that the comparison process of Stage 2 is not the same for both stimulus types. In this study, however, the slopes for letters and pictures are nearly equal. Possibly, the use of peripheral vision has the effect of raising only the slope of the function for letter stimuli. Since the theory of memory scanning assumes that the slope represents the time needed for a comparison of the test stimulus with a single memory-set element, the increased slope might indicate that the letter test stimulus representation is less "clear" with peripheral presentation than with foveal and the picture test representation remains the same. Thus, this result appears to agree with the hypothesis that letters are processed in spatial terms, whereas pictures are verbally represented and, therefore, unaffected by visual manipulations.

The present hypothesis was that, in this experiment, letter test stimuli are processed by the right, spatial, hemisphere, whereas pictures are processed by the left, verbal, hemisphere. This leads to the prediction that letters presented to the left hemisphere must cross the corpus callosum before being processed, leading to an increase in the intercept of the corresponding RT function. Similarly, pictures that enter the right hemisphere must be transferred to the left, thus raising the intercept of that function. If it is assumed that the comparison process is unaffected by callosal transmission, the slopes should be the same for right- and left-hemispheric presentation of a stimulus,

An examination of the intercepts of the RT functions for positive responses, negative responses, and both responses combined supports the hypothesis. In each case, as predicted, the intercept of the function for letter stimuli is higher for left-hemispheric presentation than for right, while the reverse holds true for pictures. The difference in intercepts for the combined data of positive and negative responses is $45.0 \mathrm{msec}$ for letters and 33.2 msec for pictures.

Also as predicted. the slope of the function for letter test stimuli presented to the right hemisphere is the same as for presentation to the left. However, this does not hold true for the case of picture stimuli. The slope of the function for picture stimuli presented to the right hemisphere is less than for presentation to the left, indicating a difference in comparison times. One possibility is that this occurs because the transfer of information from the right to the left hemisphere has a facilitative effect on the comparison process. It is also possible that presenting a picture to the left hemisphere, where comparisons are supposed to take place, makes the comparison process more difficult. A third possibility is that some picture stimuli are never transferred at all. This might occur if a picture could be equated with its corresponding letter without the aid of verbalization, enabling the right hemisphere to perform spatial comparisons when it receives a picture stimulus.

This study was intended as a preliminary investigation of the effects of interhemispheric transfer on memory scanning with the letter-and-picture paradigm. Accordingly, a more extensive

experiment is in progress, and it is hoped that that research will support and clarify the present results.

$$
\text { REFERENCES }
$$

BRADSHAW, J. L.. \& PERRIMENT, A. D. Latcrality effects and choice reaction time in a unimanual two-finger task. Perception \& Psychophysics, 1970, 7, 185-188.

FILBEY, R. A., \& GAZZANIGA, M. S. Splitting the normal brain with reaction time Psychonomic Science, 1969, 17, 335-336.

GIBSON, A. R., FILBEY, R., \& GAZZANIGA, M. S. Hemispheric differences as reflected by reaction time. Federation Proceedings, 1970, 29, 658. (Abstract)

KLATZKY, R. L., \& ATKINSON, R. C. Memory scans based on alternative test stimulus representations. Perception \& Psychophysics, $1970,8,113-117$.

KLATZKY, R. L., JUOLA, J. F., \& ATKINSON, R. C. Test stimulus representation and experimental context effects in memory scanning. Institute for Mathematical Studies in the Social Sciences, Stanford University, Technical Report No. 160, 1970.

LEVY, J. Possible basis for the evolution of lateral specialization of the human brain. Nature, 1969, 224, 614-615.

LEVY-AGRESTI, J., \& SPERRY, R. W. Differential perceptual capacities in major and minor hemispheres. Proceedings of the National Academy of Sciences, 1968, 61. 1151.

MOSCOVITCH, M., \& CATLIN, J. Interhemispheric transmission of information: Measurement in normal man. Psychonomic Science, 1970, 18, 211-212.

STERNBERG, $S$. High-speed scanning in human memory. Science, 1966, 153, 652-654.

\title{
An investigation into changes in decision criteria and other details of a decision-making task
}

\author{
M. HAMMERTON \\ Applied Psychology Unit, Cambridge, England
}

A method is described for constructing a decision-making task which satisfies the formal assumptions of signal-detection theory. Two experiments using this task are described. It is shown that Ss did not maintain a constant likelihood-ratio criterion and, hence, that the subjective $d^{\prime}$ was always less than the true value, even though Ss knew what that value was. Introducing confidence judgments did not significantly affect $d^{\prime}$. It is suggested that the method described is a useful one for examining decision responses.

The purpose of this study was to construct a decision-making task whose characteristics matched the assumptions of signal detection theory (e.g. Tanner \& Swets, 1954) with the modification that the individual presentations of evidence to $S$ of "signal" or of "signal + noise" were plain and manifest. To this end, each presentation consisted of a two-digit number drawn from a known population of numbers. In each experiment, two such populations were used, both being Gaussian; and each presentation was drawn with equal probability from either. The population regarded as "noise" had a mean of 40: the "signal" population had a mean of 43.47 . and 50 in the several experiments performed so far; and all populations had a variance of 10 .

Each number was clearly displayed to the $\mathrm{S}$, the digits being self-luminous, $2.5 \mathrm{~cm}$ high and approximately $60 \mathrm{~cm}$ from $S$ 's eyes.

It will be noted that this experimental method satisfies the formal assumptions of signal detection theory; but that such necessary uncertainties as, e.g., the exact form of the distributions, are removed; and S's evidence is precisely determined. It is

Table 1

Group Median Values of Subjective $d^{\prime}$ and $\beta$

\begin{tabular}{llll}
\hline True d' & 0.95 & 2.21 & 3.16 \\
Subjective d & 0.69 & 1.64 & 2.90 \\
$\beta$ & 1.01 & 1.10 & 0.89 \\
\hline
\end{tabular}

